\title{
Association of Cord Blood Thyroid-Stimulating Hormone Levels with Maternal, Delivery and Infant Factors
}

Karen ML Tan, ${ }^{1,2}$ PhD, MBBS, FRCPath, Anne HY Chu,${ }^{1}$ PhD, See Ling Loy, ${ }^{3,4}{ }_{P h D}$, Victor Samuel Rajadurai, ${ }^{5}$ MBBS, MD (Paed), FAMS,

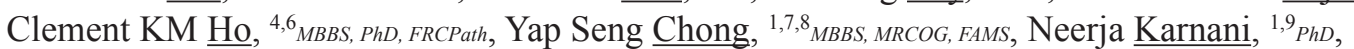

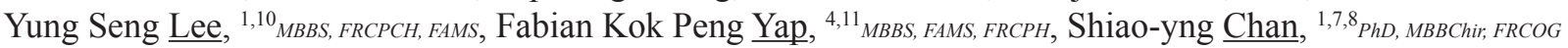

\begin{abstract}
Introduction: This study examined maternal, delivery and infant factors associated with cord thyroid-stimulating hormone (TSH) concentrations in an Asian population.

Methods: The Growing Up in Singapore Towards healthy Outcomes (GUSTO) study is a mother-offspring birth cohort from 2 major hospitals in Singapore. Cord serum TSH was measured using the Abbott ARCHITECT TSH Chemiluminescent Microparticle Immunoassay and the ADVIA Centaur TSH-3 Immunoassay. After excluding infants with a maternal history of thyroid disease, screening cord TSH results from 604 infants were available for multivariable regression analysis in relation to the factors of interest.
\end{abstract}

Results: Babies born by vaginal delivery had significantly higher cord serum TSH concentrations than babies born by caesarean section. Cord serum TSH concentrations differed significantly by measurement method. There was no association of cord TSH concentrations with ethnicity, sex, birth weight, gestational age, maternal body mass index, gestational weight gain, gestational diabetes mellitus status and other maternal, delivery and infant factors studied.

Conclusion: Interpretation of cord serum TSH results may need to take into account mode of delivery and measurement method.

Ann Acad Med Singap 2020;49:937-47

Keywords: Cord blood, Growing Up in Singapore Towards healthy Outcomes, GUSTO, perinatal, TSH

\section{Introduction}

The thyroid status of neonates is known to have a significant impact on brain development. ${ }^{1}$ Congenital hypothyroidism is one of the most common preventable causes of childhood mental retardation ${ }^{2}$ and has an estimated local incidence of about 1 in $1,000 .^{3}$ In Singapore, national screening for congenital hypothyroidism has been performed on umbilical cord serum due to an early discharge policy since $1980 .^{3}$ The screening strategy initially used cord serum thyroxine (T4) as the primary screen, followed by a strategy using both T4 and thyroid-stimulating hormone (TSH) in

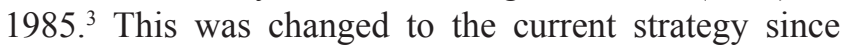
1990 using TSH as the primary screen as TSH is the critical component for sensitivity, and to reduce screening cost. ${ }^{3}$ Those exceeding the 99th percentile for $\mathrm{TSH}$ are recalled for further investigation, including free thyroxine (fT4) levels. ${ }^{3}$

\footnotetext{
${ }^{1}$ Singapore Institute for Clinical Sciences, Agency for Science, Technology and Research, Singapore

${ }^{2}$ Department of Laboratory Medicine, National University Hospital, Singapore

${ }^{3}$ Department of Reproductive Medicine, KK Women's and Children's Hospital, Singapore

${ }^{4}$ Duke-NUS Medical School, Singapore

${ }^{5}$ Department of Neonatology, KK Women's and Children's Hospital, Singapore

${ }^{6}$ Department of Pathology and Laboratory Medicine, KK Women's and Children's Hospital, Singapore

${ }^{7}$ Department of Obstetrics and Gynaecology, Yong Loo Lin School of Medicine, National University of Singapore, Singapore

${ }^{8}$ Department of Obstetrics and Gynaecology, National University Hospital, Singapore

${ }^{9}$ Department of Biochemistry, Yong Loo Lin School of Medicine, National University of Singapore, Singapore

${ }^{10}$ Khoo Teck Puat - National University Children's Medical Institute, Singapore

${ }^{11}$ Department of Paediatrics, KK Women's and Children's Hospital, Singapore

Address for Correspondence: A/Prof Shiao-yng Chan, Singapore Institute for Clinical Sciences, Agency for Science, Technology and Research, Brenner Centre for Molecular Medicine, 30 Medical Drive, Singapore 117609

E-mail: obgchan@nus.edu.sg
} 
Infant TSH and thyroid hormone levels are known to be influenced by maternal, delivery and infant factors. ${ }^{4}$ However, conflicting associations have been reported. ${ }^{4}$ For example, gestational diabetes mellitus (GDM) has been associated with higher cord blood TSH levels. ${ }^{5,6}$ However, other studies found no association between GDM and neonatal thyroid status. ${ }^{7-10} \mathrm{GDM}$ is common in Singapore and in this GUSTO cohort, $18.9 \%$ of pregnancies were affected. ${ }^{13}$ If GDM is associated with elevated cord TSH, this could have implications for reference ranges and infant follow-up. We aimed to examine whether GDM, maternal fasting and 2-hour post-oral glucose tolerance test (OGTT) glucose levels are associated with cord serum TSH levels in infants of mothers with no history of thyroid disease. We also examined other maternal, delivery and infant factors in relation to cord serum TSH levels in our multi-ethnic Asian cohort.

\section{Methods}

\section{Study design and population}

Data were obtained from the Growing Up in Singapore Towards healthy Outcomes (GUSTO) study, an Asian prospective birth cohort in Singapore. ${ }^{11}$ One thousand two hundred and forty seven pregnant women were recruited at 11-14 weeks gestation from the 2 largest public maternity hospitals in Singapore-KK Women's and Children's Hospital (KKH) and National University Hospital (NUH) - from June 2009 to September 2010. The inclusion criteria for GUSTO included age between 18 and 50 years, intention to live in Singapore for the next 5 years, intention to deliver in $\mathrm{KKH}$ and $\mathrm{NUH}$, and willingness to donate cord, cord blood and placenta.

\section{Data collection}

Maternal demographic and clinical data were collected at multiple study visits, using interviewer-administered questionnaires and hospital medical records, according to standardised protocols. Maternal smoking was defined as any smoking prior to pregnancy or during pregnancy, while socio-economic status was determined using highest education obtained. Following delivery, data on labour, mode of delivery and complications were obtained from hospital case notes by trained health personnel.

\section{Gestational age}

Gestational age (GA) was determined by ultrasonography in the first trimester. Scans were conducted by trained ultrasonographers in a standard manner at both hospitals and GA was reported in weeks completed. Preterm births were defined as births occurring at less than 37 weeks of gestation.

\section{Anthropometric measurement}

At 26-28 weeks' gestation, maternal height was measured using the SECA 213 Stadiometer (SECA Corp). Maternal weight at booking (10-11 weeks) and weight at delivery were obtained from hospital medical records. Antenatal body mass index (BMI) was calculated as booking weight $(\mathrm{kg})$ divided by the square of height (m). Gestational weight gain (GWG) was calculated as the maternal weight at delivery minus the maternal weight at booking. Measurements of infant birthweight and infant birth length were retrieved from medical records.

\section{Oral glucose tolerance testing}

Mothers were given a $75 \mathrm{~g}$ oral glucose tolerance test (OGTT) after $8-10 \mathrm{~h}$ of fasting around 26 weeks of gestation (mean \pm S.D $=26.8 \pm 2.12$ weeks). Fasting and $2 \mathrm{~h}$ post-OGTT venous blood samples were collected in fluoride tubes and plasma glucose concentrations measured using the Beckman LX20 Pro analyser (Beckman Coulter) at KKH and the Advia 2400 Chemistry analyser (Siemens) at NUH. ${ }^{13}$ Women were considered as having GDM if their fasting glucose was $\geq 7.0 \mathrm{mmol} / \mathrm{L}$ and $/$ or their $2 \mathrm{~h}$ post-OGTT glucose was $\geq 7.8 \mathrm{mmol} / \mathrm{L}$, according to the World Health Organization 1999 criteria. $^{14}$

\section{Cord serum TSH}

Cord blood was obtained at the time of birth and measured in the laboratories of the respective hospitals. Cord serum TSH concentrations were obtained from the laboratory electronic records from both hospitals. Unfortunately cord serum TSH concentrations were found in the laboratory electronic records for approximately only half of the cohort (Table 1). Cord serum TSH was measured using the 3rd generation Abbott ARCHITECT TSH Chemiluminescent Microparticle Immunoassay (CMIA) at KKH, and using the 3rd generation ADVIA Centaur TSH-3 Immunoassay at NUH. A cut-off of $25 \mathrm{mU} / \mathrm{L}$ was used at both hospitals, as the lowest screening TSH was found to be $25 \mathrm{mU} / \mathrm{L}$ among cases of congenital hypothyroidism detected. ${ }^{3}$ A total of 613 cord serum TSH concentrations were retrievable from the hospital laboratory records, 505 from $\mathrm{KKH}$ and 108 from NUH. Nine of these results were excluded from the analyses as the mothers either had a history of thyroid disease and/or were on thyroxine or anti-thyroid medications (Fig. 1). 


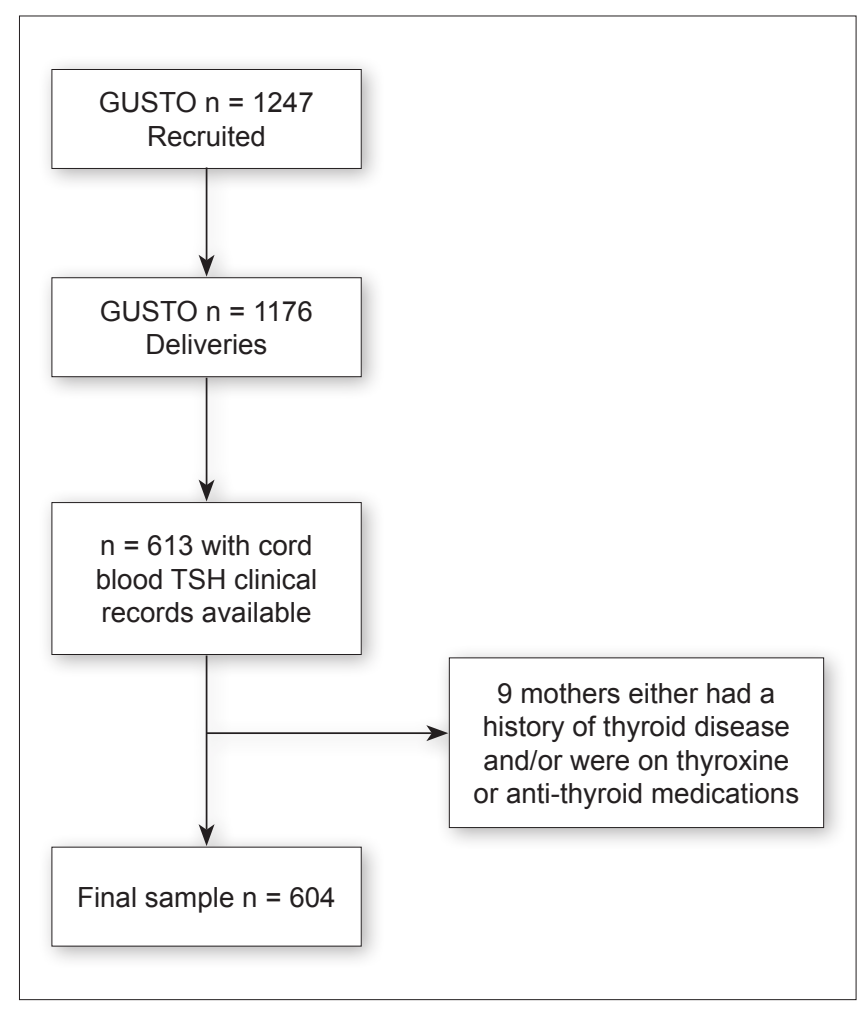

Fig. 1. Flowchart of the study population and sample size for analysis of factors associated with cord TSH.

\section{Statistical analyses}

Multivariable regression analyses were performed. Cord serum TSH was not normally distributed and therefore standardised scores of $\log _{10}$-transformed TSH were used. Covariates were controlled for based on prior knowledge from the literature about factors that might confound the associations between cord serum TSH and maternal, delivery and infant factors. Regression analyses were adjusted for hospital, ethnicity, child's sex, mode of delivery, GA, birth weight, birth order, Apgar score at 1 minute, maternal age, maternal smoking, maternal education, maternal BMI, GWG and maternal GDM status.

All statistical analyses were performed using SPSS statistics Version 20 (IBM Corp, Armonk, US). Twosided tests were used, and a value of $P<0.05$ was considered statistically significant in the univariate model while $s<0.0035(0.05 / 14)$ was considered statistically significant to correct for multiple testing (for 14 variables tested) in the multivariate model.

\section{Results}

The characteristics of the cohort stratified into the subset with cord serum TSH data available and the other without cord TSH data are shown in Table 1. More infants from $\mathrm{KKH}$ had cord serum TSH data available than those from NUH. The subset with cord serum TSH data had a lower mean gestational age, more preterm infants and fewer first-born infants compared with infants without TSH data. There was no significant difference between those with and without cord serum TSH data in ethnicity, GDM incidence, maternal BMI, maternal age, maternal smoking, maternal education, infant sex, and birthweight.

The median cord serum TSH was $5.0 \mathrm{mIU} / \mathrm{L}$ (range 0.7-40.0). Cord serum TSH was log-normally distributed. The median cord serum TSH concentration was significantly lower at KKH (Abbott Architect CMIA, median \pm interquartile range: $4.9 \pm 3.17 \mathrm{mIU} / \mathrm{L})$ compared with NUH (Advia Centaur, 5.9 $\pm 4.63 \mathrm{mIU} / \mathrm{L}$ ). A universal reference range derived from local population studies for cord serum TSH of $2.2-25.0 \mathrm{mIU} / \mathrm{L}$ is used at both hospitals. Using the laboratory reference interval upper limit of $25 \mathrm{mIU} / \mathrm{L}, 3$ infants were recalled for possible congenital hypothyroidism at $\mathrm{KKH}$ and 2 infants at NUH. Based on the 99th percentile for cord TSH derived from our own limited dataset of 604 infants, the study-derived site-specific 99th percentile of cord TSH was $20.3 \mathrm{mIU} / \mathrm{L}$ at $\mathrm{KKH}$ and $39.6 \mathrm{mIU} / \mathrm{L}$ at NUH. If study-derived site-specific 99th percentiles were used, 5 infants would be recalled at $\mathrm{KKH}$ and 1 infant from NUH.

\section{Maternal factors}

Maternal age, maternal BMI and GWG were not associated with umbilical cord serum TSH concentrations (Table 2). There was no significant difference in cord serum TSH across ethnic groups. GDM status was not associated with cord serum TSH after adjusting for hospital and other confounders, and both fasting and 2-hour post-OGTT glucose levels were also not associated with umbilical cord serum TSH concentrations (Table 2). Pre-eclampsia and pregnancyinduced hypertension were also not associated with cord serum TSH concentrations.

\section{Delivery factors}

Babies born by vaginal delivery had significantly higher cord serum TSH concentrations than babies born by caesarean section (Table 2). There was no significant difference in cord serum TSH concentrations between babies born by caesarean section conducted during labour (intrapartum) and babies born by caesarean section without labour (non-labour). Babies delivered by assisted vaginal delivery (forceps or vacuum) had higher cord serum TSH concentrations compared to babies born by spontaneous vaginal delivery; however, the difference was not significant after correction for multiple testing. 
Table 1. Maternal and offspring characteristics among participants in the GUSTO study

\begin{tabular}{|c|c|c|c|}
\hline & Subjects with cord TSH levels & Subjects without cord TSH levels & \\
\hline & $(n=604)$ & $(n=573)$ & $P$ value \\
\hline Hospital & & & $<0.001$ \\
\hline $\mathrm{KKH}$ & $497(82.3)$ & $401(70.0)$ & \\
\hline NUH & $107(17.7)$ & $172(30.0)$ & \\
\hline \multicolumn{4}{|l|}{ Maternal factors } \\
\hline Ethnicity, n (\%) & & & 0.426 \\
\hline Chinese & $334(55.3)$ & $326(57.0)$ & \\
\hline Malay & $163(27.0)$ & $136(23.8)$ & \\
\hline Indian & $107(17.7)$ & $110(19.2)$ & \\
\hline No & $465(81.9)$ & $435(80.6)$ & \\
\hline Yes & $103(18.1)$ & $105(19.4)$ & \\
\hline $\begin{array}{l}\text { Pre-eclampsia or pregnancy induced hypertension } \\
\text { No } \\
\text { Yes }\end{array}$ & $\begin{array}{l}563(93.2) \\
41(6.8)\end{array}$ & $\begin{array}{l}540(94.2) \\
33(5.8)\end{array}$ & 0.467 \\
\hline $\begin{array}{l}\text { Maternal smoking } \\
\text { No } \\
\text { Yes }\end{array}$ & $\begin{array}{l}508(86.8) \\
77(13.2)\end{array}$ & $\begin{array}{l}480(85.9) \\
79(14.1)\end{array}$ & 0.633 \\
\hline $\begin{array}{l}\text { Maternal education } \\
\text { Primary } \\
\text { Secondary } \\
\text { University }\end{array}$ & $\begin{array}{l}32(5.4) \\
371(62.8) \\
188(31.8)\end{array}$ & $\begin{array}{l}25(4.5) \\
329(59.0) \\
204(36.6)\end{array}$ & 0.214 \\
\hline Maternal BMI $\left(\mathrm{kg} / \mathrm{m}^{2}\right)$ & $23.8(4.8)$ & $23.6(4.8)$ & 0.445 \\
\hline Gestational weight gain (kg) & $11.4(4.4)$ & $11.2(4.6)$ & 0.434 \\
\hline Fasting glucose (mmol/L) & $4.3(0.5)$ & $4.4(0.5)$ & 0.559 \\
\hline 2-hour post-OGTT glucose (mmol/L) & $6.5(1.5)$ & $6.6(1.5)$ & 0.537 \\
\hline \multicolumn{4}{|l|}{ Delivery factors } \\
\hline Mode of delivery & & & 0.332 \\
\hline Vaginal delivery & $413(68.6)$ & $408(71.2)$ & \\
\hline Caesarean delivery & $189(31.4)$ & $165(28.8)$ & \\
\hline Among vaginal births & & & 0.959 \\
\hline Spontaneous & $380(8.0)$ & $375(8.1)$ & \\
\hline Assisted & $33(92.0)$ & $33(91.9)$ & \\
\hline Among caesarean sections & & & 0.141 \\
\hline Intrapartum caesarean section & $57(30.2)$ & $62(37.6)$ & \\
\hline Non-labour caesarean section & $132(69.8)$ & $103(62.4)$ & \\
\hline Labour onset & & & 0.091 \\
\hline Spontaneous & $470(78.1)$ & $470(82.0)$ & \\
\hline Induced & $132(21.9)$ & $103(18.0)$ & \\
\hline
\end{tabular}


Table 1. Maternal and offspring characteristics among participants in the GUSTO study (Cont'd)

\begin{tabular}{|c|c|c|c|}
\hline & Subjects with cord TSH levels & Subjects without cord TSH levels & \\
\hline Presentation & & & 0.175 \\
\hline Cephalic & $570(94.7)$ & $551(96.3)$ & \\
\hline Breech & $32(5.3)$ & $21(3.7)$ & \\
\hline \multicolumn{4}{|l|}{ Infant factors } \\
\hline Sex & & & 0.473 \\
\hline Male & $311(51.7)$ & $308(53.8)$ & \\
\hline Female & $291(48.3)$ & $265(46.2)$ & \\
\hline Apgar score (1 minute) $\geq 7$ & & & 0.481 \\
\hline Yes & $582(96.7)$ & $557(97.4)$ & \\
\hline No & $20(3.3)$ & $15(2.6)$ & \\
\hline Preterm (<37 weeks) & & & 0.014 \\
\hline Yes & $72(11.9)$ & $44(7.7)$ & \\
\hline No & $531(88.1)$ & $529(92.3)$ & \\
\hline Birth order & & & 0.020 \\
\hline First-born & $255(42.3)$ & $281(49.0)$ & \\
\hline Not first-born & $348(57.7)$ & $292(51.0)$ & \\
\hline GA (weeks) & $38.5(1.8)$ & $38.8(1.7)$ & 0.009 \\
\hline Birth weight (kg) & $3.1(0.5)$ & $3.1(0.5)$ & 0.481 \\
\hline Birth length (cm) & $48.4(2.4)$ & $48.4(2.8)$ & 0.794 \\
\hline Head circumference (cm) & $33.3(1.5)$ & $33.3(1.7)$ & 0.583 \\
\hline
\end{tabular}

KKH: KK Women's and Children's Hospital; NUH: National University Hospital; TSH: thyroid-stimulating hormone; BMI: body mass index; OGTT: oral glucose tolerance test; GA: gestational age

a Data shown are n (\%) for categorical variables or mean (standard deviation) for continuous variables unless otherwise stated.

${ }^{b} P$ values are based on group comparison of study participants and non-participants using t-test for continuous variables and chi-square test for categorical variables.

Spontaneous labour was associated with higher cord serum TSH concentrations compared to induced labour (Table 2); however, the difference was not significant after correction for multiple testing. There was no significant difference in cord serum TSH concentrations for babies of cephalic presentation compared with breech babies.

\section{Infant factors}

Cord serum TSH concentrations did not differ by the sex of the baby (Table 2). The majority of preterm infants in this cohort were late preterm births (median 36 weeks, range 25.9-36.9 weeks). Preterm babies born at less than 37 weeks did not have statistically significantly different cord TSH concentrations compared to term babies. Babies with Apgar scores of $<7$ at 1 minute did not have statistically significantly different cord TSH concentrations, and first-born babies did not have significantly different serum cord TSH compared to later-born babies. There were too few babies with Apgar scores of $<7$ at 5 minutes for analysis. GA, birth weight, birth length, and head circumference all did not associate with serum cord TSH concentrations (Table 2).

\section{Discussion}

Of all the factors examined, only the mode of delivery had significant effects on cord serum TSH concentrations. Serum TSH concentrations are known to increase in response to stress. ${ }^{15}$ The significant association between increased cord serum TSH concentrations and vaginal birth as opposed to caesarean birth may reflect an acute response to stress experienced during labour and passage 


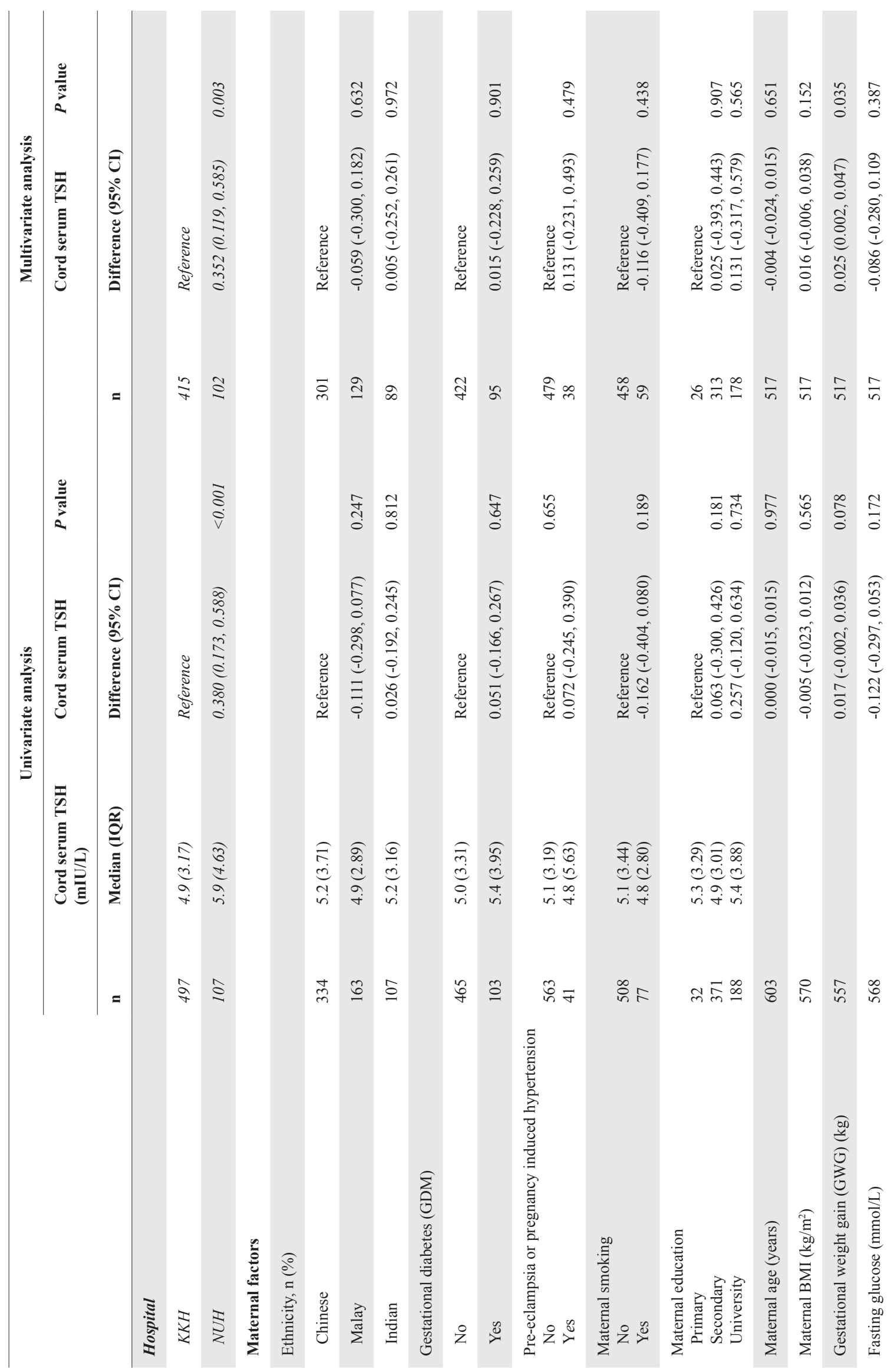




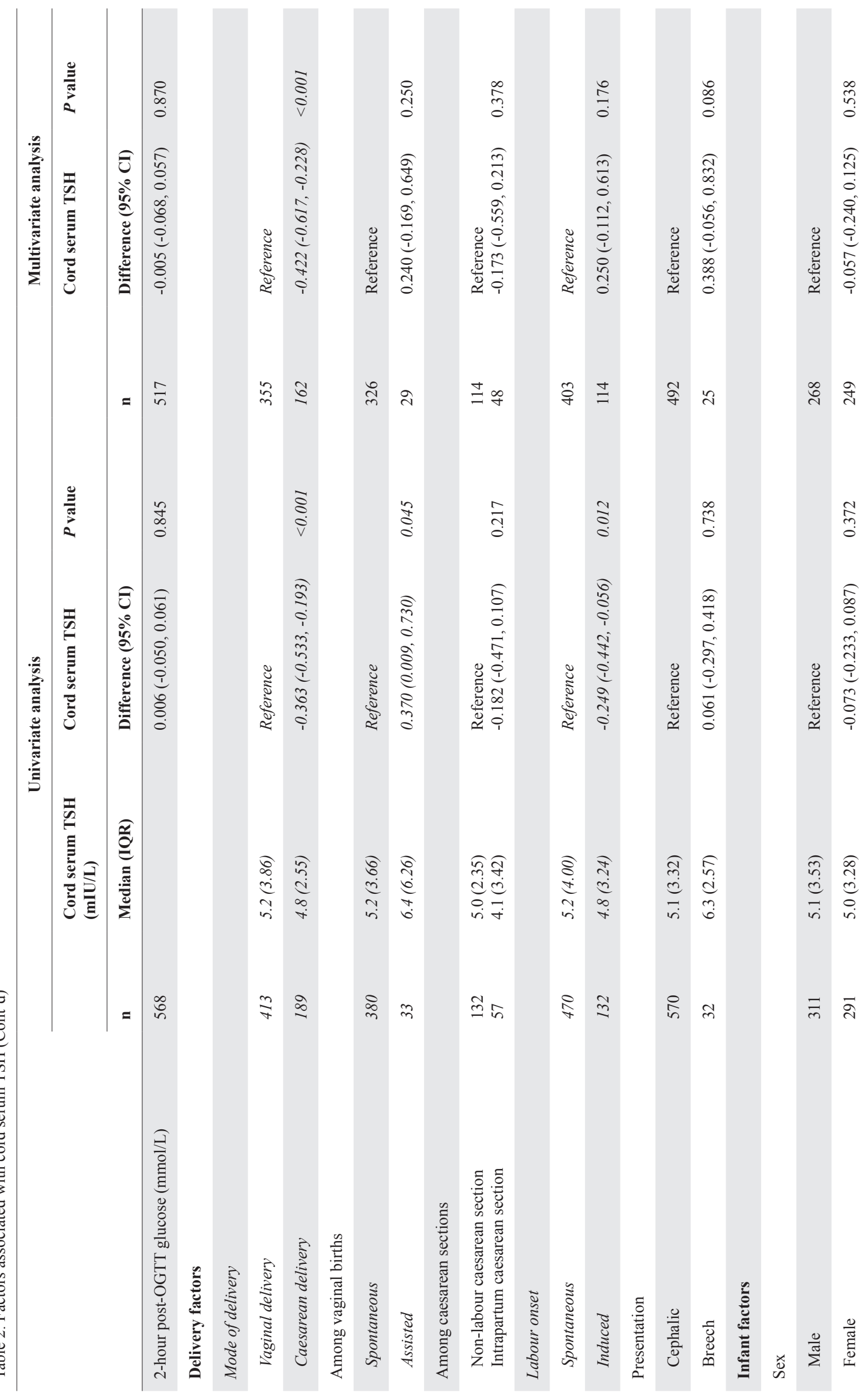




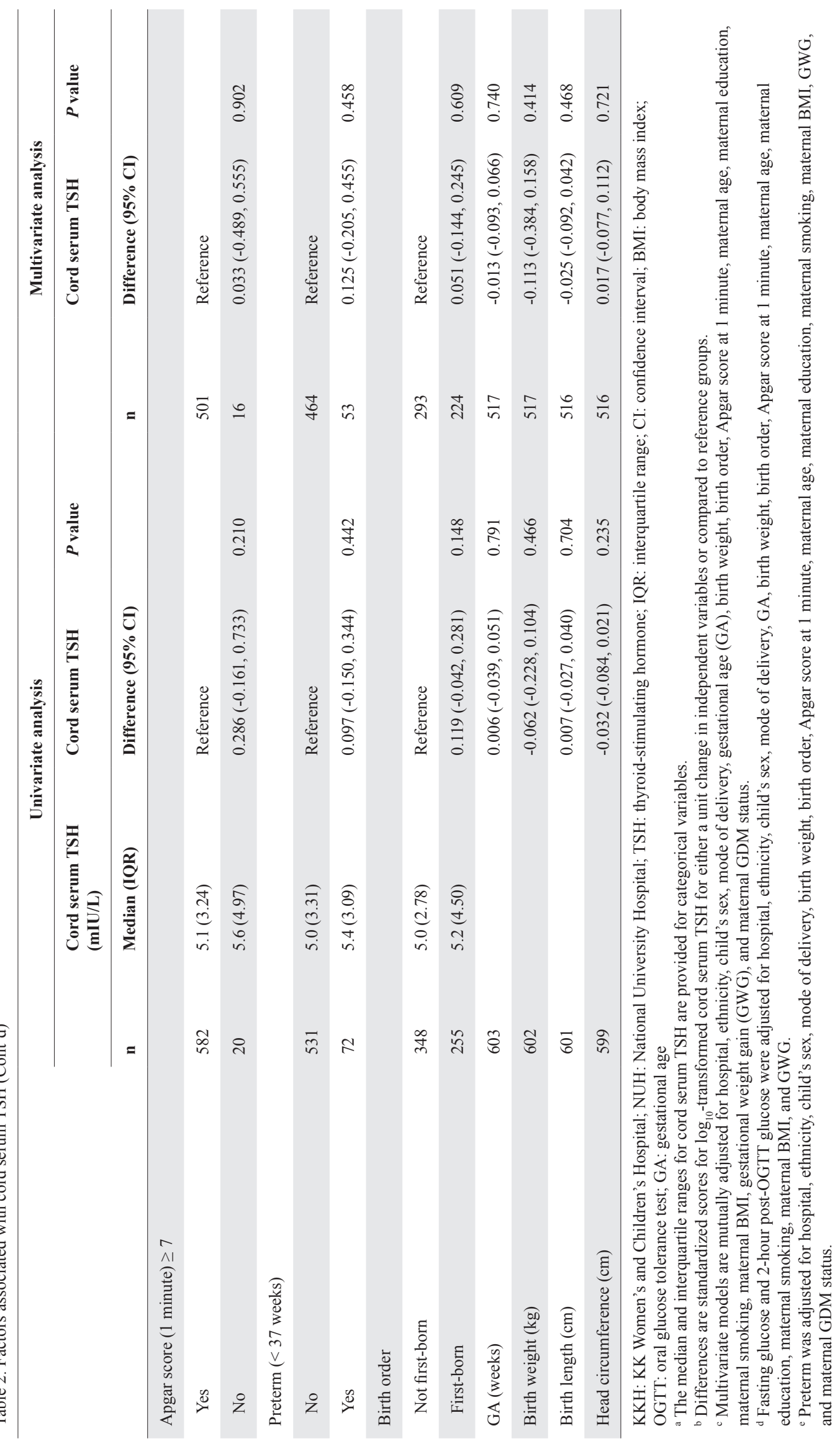


through the birth canal. We showed no significant difference in cord TSH levels between babies born by caesarean section conducted during labour and those born by caesarean section without experiencing the process of labour, indicating that it is likely the complete passage through the birth canal rather than labour itself that drives the increase in cord TSH levels. Studies have shown an association of neonatal cord TSH concentrations with perinatal stress including those associated with labour. ${ }^{16,17}$ Unfortunately data on cord blood acidosis and TSH concentrations at 2 weeks after birth are not available for comparison.

The prevalence of increased risk of congenital hypothyroidism using the local reference range was $0.8 \%$ in our cohort. This is similar to previously reported recall rates for Singapore newborn congenital hypothyroidism screening. ${ }^{3}$ Worldwide recall rates vary widely from $0.01 \%$ to $13.3 \%$ and this may be due to differing screening protocols, laboratory methods, cut-off levels, and iodine status of the population. ${ }^{19}$ Using the reference range without taking into account the method of measurement of TSH may result in 2 infants $(0.3 \%)$ missed for recall from 1 site $(\mathrm{KKH})$, therefore reference ranges may need to take into account the laboratory method. Although the use of site-specific ranges would not significantly change the total number of recalls across the 2 sites, the ones most likely to have pathology may be identified more readily for follow-up. The large difference in cord TSH conentrations between the 2 hospitals may be due to differences in measurement method and perhaps also timing and method of cord blood sampling as there was no standardised protocol across the 2 hospitals. TSH assays are not standardised, and method differences in TSH have been described, for example, using 2013 external QA data, the consensus mean TSH for sample 18 was reported as 14.8 on the ARCHITECT and 16.0 on the Centaur platform ${ }^{12}$. To address the issue of potential confounding with including both assays, we performed a sensitivity analysis using data from only KKH and similar results for association with maternal, delivery and infant factors were obtained.

\section{Maternal factors}

Asian race has been associated with higher cord TSH levels. ${ }^{9}$ This study compared TSH concentrations in cord blood among 3 different Asian ethnicities-Chinese, Malay and Indian-and did not find any significant difference. A previous local study also found no influence of ethnicity on cord TSH levels. ${ }^{38}$ Although a number of studies did not find any association between maternal diabetes and cord TSH concentrations, ${ }^{7-10}$ a limitation of these studies is that the analyses were univariate and did not adjust for potential confounding variables. In a case-control study of 469 diet-controlled GDM pregnancies that were compared with 474 non-diabetic pregnancies, higher cord TSH concentrations were found in pregnancies with GDM. ${ }^{5}$ In this study of around 600 newborns, both univariate and multivariate analyses adjusted for potential confounding variables, did not show a significant association between cord TSH concentrations and GDM, nor fasting or $2 \mathrm{~h}$ post- glucose load in an OGTT. We also did not find any association between maternal BMI or GWG and cord TSH concentrations in our cohort. Kahr et al. ${ }^{20}$ noted an association between maternal obesity with increased cord TSH concentrations, but only in very obese women, suggesting that this association may only apply at the extremes of maternal weight. However, our cohort had a mean maternal BMI of $23.8 \mathrm{~kg} / \mathrm{m}^{2}$, with only $2.8 \%$ having a $\mathrm{BMI}>35 \mathrm{~kg} / \mathrm{m}^{2}$.

\section{Delivery factors}

In literature, delivery mode has the strongest and most consistent relationship with cord TSH status. ${ }^{9}$ Most studies concluded that vaginal deliveries resulted in higher cord TSH levels compared with deliveries via caesarean section, , 16,18,21-24 consistent with the idea that stress during labour and vaginal delivery are each associated with elevated TSH in cord blood. Others reported that infants born by vacuum assisted vaginal delivery had higher cord TSH levels than infants born by spontaneous vaginal delivery. ${ }^{10}$ In this study, the cord TSH levels in infants born by forceps and vacuum assisted vaginal delivery were higher than those born by normal vaginal delivery. One study, however, reported that babies born by vaginal delivery had lower TSH compared to those born by caesarean delivery, and further, with those born by elective caesarean delivery having higher cord TSH levels compared to those born by emergency caesarean delivery, ${ }^{25}$ implying the role of labour in influencing cord serum TSH changes. In contrast, our study found no difference between cord TSH concentrations from babies born by elective caesarean section compared with those from emergency caesarean section. One study reported elevated levels of cord blood TSH in babies born vaginally after a successful external cephalic version. ${ }^{30}$ In our study there was no statistical significant difference between cord TSH levels from babies with cephalic presentation compared with those with breech presentation at birth. It is possible that the previously reported cord TSH changes may relate to the external cephalic version procedure rather than the fetal presentation. Although stress during delivery and cord blood acidosis at birth 
have been shown to be associated with elevated cord TSH concentrations, ${ }^{31}$ our study did not find a difference in cord TSH concentrations in babies with a low Apgar score at 1 minute, which could be due to small numbers and lack of statistical power.

\section{Infant factors}

We found neither an association between cord TSH levels and categorically defined preterm infants nor with GA as a continuous variable. Most studies reported no association between GA and cord TSH concentrations, ${ }^{10,16,18,22}$ while one study reported a decrease in cord TSH with increasing $\mathrm{GA}^{23}$ and another reported an increased cord TSH concentration in preterm neonates. ${ }^{9}$ At birth, term infants experience a surge in TSH that peaks around 30 minutes post-delivery. ${ }^{26}$ In very preterm infants (24-27 weeks), the hypothalamic-pituitarythyroid axis development is more immature and the TSH surge is smaller. ${ }^{27}$ Some researchers have recommended cord TSH reference ranges based on GA. ${ }^{28}$ Our study did not include very preterm infants - only 2 infants were born before 30 weeks of gestation-therefore we are unable to address the issue of using gestation-specific cord TSH reference ranges in very preterm babies for congenital hypothyroidism screening.

Birth weight has previously been reported to be positively correlated with cord TSH concentrations independently of GA. ${ }^{9}$ One study reported a decline in cord TSH concentrations with an increase in birth weight, concurrently with decreasing TSH with increasing GA. ${ }^{23}$ In contrast, Chan et al. found no association between cord TSH concentrations and birth weight after adjusting for GA. ${ }^{24}$ In agreement with our findings, other studies also reported no association between birth weight and cord TSH concentrations. ${ }^{10,18,22,29}$ Some studies reported that male neonates have higher TSH concentrations than female neonates, ${ }^{9,22,24}$ while others did not find a difference between the sexes, ${ }^{10,18,23}$ which is consistent with our study. Several studies reported increased cord TSH concentrations in first-born neonates compared to later-born neonates, ${ }^{10,22,24}$ however, we found no difference after multivariable analyses.

\section{Limitations}

One limitation of our study is that there are no measures of iodine status, which may affect thyroid hormone concentrations in pregnant women and umbilical cord TSH concentrations. ${ }^{32}$ The median urinary iodine concentration in a sample of parturient women in Singapore was found to be $165 \mu \mathrm{g} / \mathrm{L}$ a decade ago, ${ }^{33}$ which indicates iodine repletion. Recent studies have reported an association between exposure to environmental pollutants, endocrine disrupting chemicals such as polychlorinated biphenyls, and cord TSH concentrations. ${ }^{34-37}$

Another limitation of our study is that we do not have information on maternal exposure to environmental pollutants. Further, there was no measurement of neonatal thyroid volumes by ultrasonography.

Other limitations include this study being a crosssectional retrospective study over a 15 -month period with a small sample size divided between 2 sites using different methods of TSH analysis. Furthermore, there was no case of congenital hypothyroidism in the GUSTO cohort. Nation-wide screening for congenital hypothyroidism screening was initiated in the early 1990s when studies demonstrated an incidence of 3 per 1,000 deliveries. ${ }^{3,39}$

The relatively short period of data collection with the absence of any cases of congenital hypothyroidism in this study may be a limiting factor in the data interpretation and conclusion.

Our study is unique in that we have 3 different ethnic groups in Singapore. This is especially significant as Singapore uses cord serum TSH as a first line screening for congenital hypothyroidism. Although we were not able to study all variables in relation to cord TSH levels, we have studied many variables, particularly maternal factors, which have thus far been less well studied. ${ }^{9}$

In summary, from this study, we conclude that maternal glucose concentrations are not associated with cord serum TSH. Interpretation of cord serum TSH results may need to take into account the mode of delivery due to an elevation of cord TSH, possibly from the stress of passage through the birth canal.

\section{Disclosure}

Yap Seng Chong and Yung Seng Lee have received reimbursement for speaking at conferences sponsored by companies selling nutritional products. Yap Seng Chong, and Shiao-Yng Chan are part of an academic consortium that has received research funding from Abbot Nutrition, Nestec and Danone. The other authors have no conflicts of interest to declare.

\section{Acknowledgement}

The authors would like to thank the GUSTO study group.

\section{REFERENCES}

1. Chan S, Kilby MD. Thyroid hormone and central nervous system development. J Endocrinol 2000;165:1-8.

2. Léger J, Olivieri A, Donaldson M, et. al. Congenital hypothyroidism consensus conference group. European Society for Paediatric Endocrinology consensus guidelines on screening, diagnosis, and 
management of congenital hypothyroidism. Horm Res Paediatr 2014;81:80-103.

3. Joseph R, Aw TC, Tan KL. Free thyroxine as a supplement to thyrotropin in cord screening for hypothyroidism. Ann Acad Med Singap 1993;22:549-52.

4. Herbstman J, Apelberg BJ, Witter FR, et al. Maternal, infant, and delivery factors associated with neonatal thyroid hormone status. Thyroid 2008;18:67-76.

5. Lao TT, Lee CP. Gestational diabetes mellitus and neonatal hyperthyrotropinemia. Gynecol Obstet Invest 2002;53:135-9.

6. Low LC, Lin HJ, Cheung PT, et al. Screening for congenital hypothyroidism in Hong Kong. Aust Paediatr J 1986;22:53-6.

7. Wilker RE, Fleischman AR, Saenger P, et al. Thyroid hormone levels in diabetic mothers and their neonates. Am J Perinatol 1984;1:259-62.

8. Ward LS, Kunii IS, de Barros Maciel RM. Thyroid stimulating hormone levels in cord blood are not influenced by non-thyroidal mothers' diseases. Sao Paulo Med J 2000;118:144-7.

9. Herbstman J, Apelberg BJ, Witter FR, et al. Maternal, infant, and delivery factors associated with neonatal thyroid hormone status. Thyroid 2008;18:67-76.

10. Lee SY. Perinatal factors associated with neonatal thyroidstimulating hormone in normal newborns. Ann Pediatr Endocrinol Metab 2016;21:206-211

11. Soh SE, Tint MT, Gluckman PD, et al. Cohort profile: Growing Up in Singapore Towards healthy Outcomes (GUSTO) birth cohort study. Int J Epidemiol 2014;43:1401-9.

12. Clerico A, Ripoli A, Zucchelli GC, et al. Harmonization protocols for thyroid stimulating hormone (TSH) immunoassays: different approaches based on the consensus mean value. Clin Chem Lab Med 2015;53:377-82.

13. Chong YS, Cai S, Lin H, et al. Ethnic differences translate to inadequacy of high-risk screening for gestational diabetes mellitus in an Asian population: a cohort study. BMC Pregnancy Childbirth $2014 ; 14: 345$.

14. Alberti KG, Zimmet PZ. Definition, diagnosis and classification of diabetes mellitus and its complications. Part 1: diagnosis and classification of diabetes mellitus provisional report of a WHO consultation. Diabet Med 1998;15:539-53.

15. Richter SD, Schürmeyer TH, Schedlowski M, et al. Time kinetics of the endocrine response to acute psychological stress. J Clin Endocrinol Metab 1996;81:1956-60.

16. Leung WC, Chan KK, Lao TT. Neonatal hyperthyrotropinemia in gestational diabetes mellitus and perinatal complications. Neuroendocrinology 2004;80:124-8.

17. Gemer O, Shenhav S, Segal S, et al. Thyroid hormone levels in cord blood of infants with acidemia at birth. Eur J Obstet Gynecol Reprod Biol 2000;93:53-5.

18. Armanian AM, Hashemipour M, Esnaashari A, et al. Influence of perinatal factors on thyroid stimulating hormone level in cord blood. Adv Biomed Res 2013;2:48.

19. Mehran L, Khalili D, Yarahmadi S, et al. Worldwide recall rate in newborn screening programs for congenital hypothyroidism. Int $\mathrm{J}$ Endocrinol Metab 2017;15:e55451.

20. Kahr MK, Antony KM, DelBeccaro M, et al. Increasing maternal obesity is associated with alterations in both maternal and neonatal thyroid hormone levels. Clin Endocrinol (Oxf) 2016;84:551-7.

21. Franklin RC, Carpenter LM, O'Grady CM. Neonatal thyroid function: influence of perinatal factors. Arch Dis Child 1985; $60: 141-4$
22. Gupta A, Srivastava S, Bhatnagar A. Cord blood thyroid stimulating hormone level--interpretation in light of perinatal factors. Indian Pediatr 2014;51:32-6.

23. Rashmi, Seth A, Sekhri T, et al. Effect of perinatal factors on cord blood thyroid stimulating hormone levels. J Pediatr Endocrinol Metab 2007;20:59-64.

24. Chan LY, Chiu PY, Lau TK. Cord blood thyroid-stimulating hormone level in high-risk pregnancies. Eur J Obstet Gynecol Reprod Biol 2003;108:142-5.

25. Ramezani Tehrani F, Aghaee M, Asefzadeh S. The comparison of thyroid function tests in cord blood following cesarean section or vaginal delivery. Int J Endocrinol Metab 2003;1:22-26

26. Fisher DA, Klein AH. Thyroid development and disorders of thyroid function in the newborn. N Engl J Med 1981;304:702-12

27. Murphy N, Hume R, van Toor H, et al. The hypothalamic-pituitarythyroid axis in preterm infants; changes in the first 24 hours of postnatal life. J Clin Endocrinol Metab 2004;89:2824-31.

28. Hashemipour M, Hovsepian S, Ansari A, et al. Screening of congenital hypothyroidism in preterm, low birth weight and very low birth weight neonates: A systematic review. Pediatr Neonatol 2018;59:3-11.

29. Nazeri P, Shariat M, Mehrabi Y,et al. Is there an association between thyrotropin levels within the normal range and birth growth parameters in full-term newborns? J Pediatr Endocrinol Metab 2018;31:1001-1007.

30. Cha LYS, Lau TK, Chiu PY, et al. Levels of cord blood thyroid stimulating hormone after external cephalic version. BJOG 2001;108:1076-80.

31. Chan LY, Fok WY, Sahota D, et al. Cord blood thyroidstimulating hormone level and risk of acidosis at birth. Eur J Obstet Gynecol Reprod Biol 2006;124:173-7.

32. Nazeri P, Mirmiran P, Kabir A, et al. Neonatal thyrotropin concentration and iodine nutrition status of mothers: a systematic review and meta-analysis. Am J Clin Nutr 2016; 104:1628-1638.

33. Min C. Iodine status in parturient mothers and cord TSH levels in the newborn in Singapore, 5 May 2005. Available at: http:// scholarbank.nus.edu.sg/handle/10635/14640. Accessed on 12 October 2020

34. de Cock M, de Boer MR, Govarts E, et al. Thyroid-stimulating hormone levels in newborns and early life exposure to endocrinedisrupting chemicals: analysis of 3 European mother-child cohorts. Pediatr Res 2017;82:429-437.

35. Herbstman JB, Sjödin A, Apelberg BJ, et al. Birth delivery mode modifies the associations between prenatal polychlorinated biphenyl (PCB) and polybrominated diphenyl ether (PBDE) and neonatal thyroid hormone levels. Environ Health Perspect 2008;116:1376-82.

36. Itoh S, Baba $\mathrm{T}$, Yuasa $\mathrm{M}$, et al. Association of maternal serum concentration of hydroxylated polychlorinated biphenyls with maternal and neonatal thyroid hormones: The Hokkaido birth cohort study. Environ Res 2018;167:583-590.

37. Romano ME, Webster GM, Vuong AM, et al. Gestational urinary bisphenol A and maternal and newborn thyroid hormone concentrations: the HOME study. Environ Res 2015;138:453-60.

38. Joseph R, Ho LY, Gomez JM, et al. Non isotopic cord blood serum screening for congenital hypothyroidism in Singapore - the TSH and T4 strategy. In 'Neonatal Screening in the Nineties' Eds. Wilcken B, Webster D. Manly Vale, New South Wales, Australia: 8th International Neonatal Screening Symposium, 1991; 69-70.

39. Joseph R. Newborn screening in Singapore. Southeast Asian J Trop Med Public Health 1999;30 Suppl 2:23-4. 